\title{
Adhesion of vesicles in two dimensions
}

\author{
Udo Seifert* \\ Sektion Physik der Universität München, Theresienstrasse 37, 8000 München 2, \\ Federal Republic of Germany \\ (Received 14 August 1990)
}

\begin{abstract}
The adhesion of vesicles in two dimensions is studied by solving the shape equations that determine the state of lowest energy. Two ensembles are considered where for a fixed circumference of the vesicle either a pressure difference between the exterior and the interior is applied or the enclosed area is prescribed. First, a short discussion of the shape of free vesicles is given. Then, vesicles confined to a wall by an attractive potential are considered for two cases: (i) For a contact potential, a universal boundary condition determines the contact curvature as a function of the potential strength and the bending rigidity. Bound shapes are calculated, and an adhesion transition between bound and free states is found, which arises from the competition between bending and adhesion energy. (ii) For adhesion in a potential with finite range, the crossover from the longranged to the short-ranged case is studied. For a short-ranged potential, a decrease in the strength of the potential can lead to a shape transition between a bound state and a "pinned" state, where the vesicle acquires its free shape but remains pinned by the potential. In such a potential, fluctuations lead to unbinding for which two different cases are found. Small vesicles unbind via fluctuations of their position, while large vesicles unbind via shape fluctuations.
\end{abstract}

\section{INTRODUCTION}

Vesicles are closed bilayers of lipid molecules ${ }^{1}$ and can theoretically be characterized as two-dimensional (2D) surfaces embedded in three-dimensional space. Their shape is primarily determined by bending energy, i.e., by curvature. $^{2}$ Several theoretical studies ${ }^{3-5}$ have been devoted to an investigation of these shapes as a function of the surface area and enclosed volume or applied (osmotic) pressure difference. If one ignores fluctuations, these shapes are determined by minimizing a free-energy functional. Even for the restricted set of axisymmetric shapes, a surprising variety of solutions to the corresponding shape equations have been found, and the systematic phase diagram has only recently been obtained. ${ }^{6}$ Nonaxisymmetric shapes and the influence of fluctuations have been studied for small perturbations around the spherical shape. ${ }^{7,8}$ Simulations of fluid vesicles are very time consuming and only recently have results become available. ${ }^{9}$ In experiments, shape transformations have indeed been seen by varying the temperature, ${ }^{10,11}$ i.e., the area of the vesicle. A comparison of such an experiment with theoretical calculations shows good agreement. ${ }^{11}$ An analysis of thermally excited shape fluctuations allows the determination of the bending rigidity quantitatively. ${ }^{12}$

As far as biological and biophysical applications are concerned, studies of the interaction of vesicles with membranes or other vesicles are important. Recently a simple theoretical model for the adhesion of vesicles was introduced and studied that led to various bound states and an adhesion transition that is driven by the competition between adhesion and bending energies. ${ }^{13,14}$ This transition already appears at the level of the solutions of minimal energy and is thus different from the unbinding transition of planar membranes driven by shape fluctuations. ${ }^{15-17}$

Given the complex behavior of vesicles in three dimensions, it is useful to simplify the problem by reducing the dimension and to study closed one-dimensional loops or vesicles embedded in two-dimensional space. ${ }^{18,19}$ The shape of these vesicles in two dimensions is determined by the curvature energy of their contour under the constraint of fixed enclosed area or an applied pressure difference between the exterior and interior. These alternatives define the $A$ and $P$ ensembles. The reduction in dimension is especially effective for simulations that have been performed in the $P$ ensemble for free vesicles ${ }^{20,21}$ and vesicles confined to a wall by a linear potential. ${ }^{22}$ Different scaling regimes have been found in these studies. A crucial quantity for the characterization of the typical configuration is the persistence length $L_{\kappa}$, which measures the length over which the orientation of the vesicle's contour is correlated. In two dimensions $L_{\kappa}$ is given by

$$
L_{\kappa} \equiv \kappa / T
$$

where $\kappa$ denotes the bending rigidity of dimension energy times length, while $T$ is the temperature measured in units of Boltzmann's constant. If the linear size $R$ of the vesicle is large compared to $L_{\kappa}$, the bending energy becomes irrelevant and the vesicle behaves like a closed self-avoiding walk.

For $R \ll L_{\kappa}$, however, the bending energy is relevant and determines the shape together with the constraint on the enclosed area or applied pressure difference. It is this latter stiff regime that is important in relation to the three-dimensional problem, since in three dimensions the 
persistence length is exponential in $\kappa / T$ and typically much larger than the size of the vesicles. In this regime, thermal fluctuations are relatively small and the real shapes can well be approximated by the shapes of lowest energy. These shapes will be determined in this paper for two cases: (i) for free vesicles and (ii) for vesicles adhering to a wall.

Compared to the three-dimensional case, free vesicles in two dimensions exhibit two simplifying aspects: (i) the spontaneous curvature has only a trivial effect ${ }^{18}$ and (ii) the difference between oblate and prolate ellipsoids of revolution is missing. Consequently the phase diagram, which is determined by the shape of lowest energy, is fairly simple. I will describe it in Sec. II since it will form the basis for the study of adhering vesicle.

In Sec. III, the adhesion of vesicles to a contact potential is studied in detail. One finds a rather universal boundary condition for the contact curvature and the occurrence of an adhesion transition between bound- and free-vesicle states. Both features arise from the competition of bending and adhesion energy. I discuss this transition which can be of first or second order in both ensembles, and compare it with the three-dimensional case. $^{13,14}$

In Sec. IV, adhesion in a potential with finite range is considered. ${ }^{23}$ The shape equations are derived for an arbitrary potential and discussed for a two-parameter model potential. In such a potential the state of lowest energy is always bound. The crossover from a long-ranged to the short-ranged case is studied. For the latter case, the adhesion transition found in the presence of a contact potential case corresponds to a transition between a bound and a "pinned" state. In the latter state, the vesicle is merely pinned by the potential but acquires its free shape.

The influence of fluctuations is studied qualitatively in Sec. V for $L_{\kappa} \gg R$. These fluctuations lead to an unbinding of the vesicle in a finite-range potential and introduce a new length scale $R_{c}$. If the strength of the potential decreases, two different cases must be distinguished, depending on the linear size $R$ of the vesicle. Vesicles with $R<R_{c}$ unbind via fluctuations of their position, while the unbinding of large vesicles with $R>R_{c}$ is driven by shape fluctuations.

\section{FREE VESICLE}

\section{A. Shape equation}

I consider free vesicles with a constant contour length $L \equiv 2 \pi R$. The shape of a vesicle in the continuum description can be parametrized by $C(S)$, where $C$ is the local curvature and $S$ the length along the contour. The bending energy $F_{\kappa}$ is then given by

$$
F_{\kappa} \equiv(\kappa / 2) \int_{0}^{2 \pi R} d S\left[C(S)-C_{0}\right]^{2} .
$$

$\kappa$ is the $2 \mathrm{D}$ bending rigidity with dimension energy times length and $C_{0}$ denotes a spontaneous curvature that will not enter the shape equations as shown below. ${ }^{18}$

Two different ensembles have to be distinguished. In the $P$ ensemble, a two-dimensional pressure difference $P$ between the exterior and the interior of the vesicle is ap- plied. Shape transformations then involve the enthalpy term

$$
F_{P} \equiv P A
$$

where $A$ is the enclosed area. In the $A$ ensemble, this area

$$
A \equiv \pi R^{2}(1-\epsilon)
$$

is prescribed and defines $\epsilon$. In this case, $P$ is a Lagrange multiplier which has to be determined afterward in order to ensure this prescribed area.

In both cases, the shapes of lowest energy are among the stationary points of the free energy

$$
F \equiv F_{\kappa}+F_{P} \text {. }
$$

It is convenient to scale the contour length $L$ of the vesicle to $2 \pi$, which leads to

$$
F=(\kappa / R)\left[\int_{0}^{2 \pi} d s \frac{1}{2}\left[c(s)-c_{0}\right]^{2}+\frac{p A}{R^{2}}\right] .
$$

Here

$$
p \equiv P R^{3} / \kappa
$$

is a scaled pressure, $c_{0} \equiv C_{0} R$ the scaled spontaneous curvature, and $s \equiv S / R$ the arc length along the rescaled contour. The scaled shape is parametrized in two different ways, namely $\psi(s)$ and $\rho(\theta)$ as shown in Fig. 1. For a numerical study of the shape equations, the parametrization $\psi(s)$ is more convenient, while $\rho(\theta)$ is appropriate for the analytical stability analysis of the circular vesicle as given further below. I restrict my search for extremal solutions to those that have at least one axis of symmetry which is denoted as the $z$ axis. The coordinates $r$ and $z$ then obey

$$
\begin{aligned}
& \dot{z}=-\sin \psi, \\
& \dot{r}=\cos \psi,
\end{aligned}
$$

where the dot denotes a derivative with respect to the arc length $s$. The free energy (2.5) can now be written

$$
F=(2 \kappa / R) \int_{0}^{\pi} d s\left[\frac{1}{2}\left(\dot{\psi}-c_{0}\right)^{2}+p r \sin \psi+\gamma(\dot{r}-\cos \psi)\right] .
$$

The last term with the Lagrange multiplier function $\gamma(s)$ has to be introduced because $r$ and $\psi$ are mutually dependent via Eq. (2.7b). The complete set of Euler-Lagrange

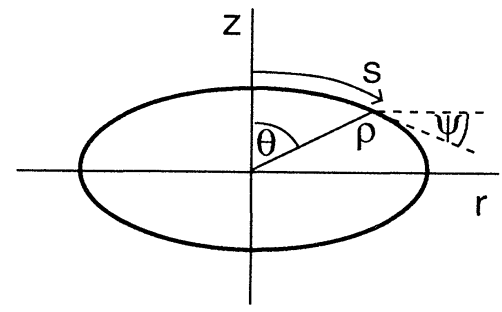

FIG. 1. Parametrization of the contour of the vesicle. 
(EL) equations for the free energy (2.8) reads

$$
\begin{aligned}
& \dot{\psi}=u, \\
& \dot{u}=p r \cos \psi+\gamma \sin \psi, \\
& \dot{\gamma}=p \sin \psi, \\
& \dot{r}=\cos \psi .
\end{aligned}
$$

The boundary conditions are

$$
\begin{aligned}
& \psi(0)=0, \\
& \psi(\pi)=\pi, \\
& r(0)=0, \\
& r(\pi)=0 .
\end{aligned}
$$

Note that the spontaneous curvature does not enter the shape equations. ${ }^{18}$ Its constant contribution to $F$ will be omitted in the rest of the paper. Although these equations look simple, an analytic solution is not available in general.

\section{B. Stability analysis of the circular shape}

For $p=0$, it can be shown that the circle $\psi(s) \equiv s$ is the only solution. First, observe that $\gamma$ is constant in this case. Then $\psi$ obeys the "mechanical" equation of motion $\ddot{\psi}=-\partial V / \partial \psi$, with $V(\psi)=\gamma \cos \psi$. For $\gamma \neq 0$, any solution of this equation with $\psi(0)=0$ and $\psi(\pi)=\pi$ will lead to $\int_{0}^{\pi} \cos \psi(s) d s \equiv r(\pi)-r(0) \neq 0$, which contradicts the boundary conditions for $r$. Thus $\gamma=0$ and $\psi(s)=s$. It is easy to verify that this circle is indeed a solution for any $p$. It does not, however, correspond to a minimum of $F$ in the $P$ ensemble for arbitrary $p$. This can be investigated by a stability analysis of this circular solution as follows.

Consider a harmonic perturbation of the circle

$$
\begin{aligned}
& \bar{\rho}(\theta) \equiv R_{\rho}(\theta) \\
& =R\left[1+a_{0}+\sum_{k(\geq 1)}\left(a_{k} \cos k \theta+b_{k} \sin k \theta\right] .\right.
\end{aligned}
$$

This vesicle has a contour length

$$
\begin{aligned}
L & =R \int_{0}^{2 \pi} d \theta\left(\rho^{2}+\rho^{\prime 2}\right)^{1 / 2} \\
& \approx 2 \pi R\left(1+a_{0}+\sum_{k(\geq 1)}\left(k^{2} / 4\right)\left(a_{k}^{2}+b_{k}^{2}\right)\right],
\end{aligned}
$$

up to second order in $a_{k}$ and $b_{k}$, where the prime denotes a derivative with respect to $\theta$. Since $L=2 \pi R$ is fixed, one finds

$$
a_{0} \approx-\sum_{k(\geq 1)}\left(k^{2} / 4\right)\left(a_{k}^{2}+b_{k}^{2}\right) .
$$

Clearly, the fluctuations absorb the length, and the mean radius $R\left(1+a_{0}\right)$ of a noncircular shape is smaller than that of a circle with the same contour length. The area $A$ of the deformed vesicle is given by

$$
\begin{aligned}
A & =R^{2} \int_{0}^{2 \pi} d \theta \rho^{2} / 2 \\
& \approx R^{2} \pi\left[1-\frac{1}{2} \sum_{(k \geq 1)}\left(k^{2}-1\right)\left(a_{k}^{2}+b_{k}^{2}\right)\right]
\end{aligned}
$$

and its curvature energy as

$$
\begin{aligned}
F_{\kappa} & =(\kappa / R) \int_{0}^{2 \pi} d \theta \frac{1}{2}\left(\rho^{2}-2 \rho^{\prime 2}-\rho \rho^{\prime \prime}\right)^{2} /\left(\rho^{2}+\rho^{\prime 2}\right)^{5 / 2} \\
& \approx(\kappa / R) \pi\left[1+\frac{1}{2} \sum_{k(\geq 1)}\left(k^{2}-1\right)^{2}\left(a_{k}^{2}+b_{k}^{2}\right)\right] .
\end{aligned}
$$

From (2.14) and (2.15), I find for the free energy

$$
\begin{gathered}
F \approx(\kappa / R) \pi\left(1+p+\frac{1}{2} \sum_{k(\geq 1)}\left(k^{2}-1\right)\left(k^{2}-1-p\right)\right. \\
\left.\times\left(a_{k}^{2}+b_{k}^{2}\right)\right] .
\end{gathered}
$$

This result shows that the $k$ mode becomes a soft mode at

$$
p_{c}(k) \equiv k^{2}-1 \text {. }
$$

Moreover, the second-order contribution to $F$ vanishes for any $p$ and $k=1$, which corresponds to a translation.

For $p<3=p_{c}(2) \equiv p_{2}$, the circle is locally stable. At $p=p_{2}$, it becomes unstable with respect to the elliptical $k=2$ deformation. A calculation to $O\left(a_{2}^{4}\right)$ reveals that the term $O\left(a_{2}^{4}\right)$ is indeed positive for $p \gtrsim p_{2}$, which leads to $a_{2} \simeq \pm\left(p-p_{2}\right)^{1 / 2}$ for $p \geq p_{2}$. Thus the shape for $p \gtrsim p_{2}$ evolves continuously from the circular shape at this second-order transition $\left(C_{f}\right)$. Note that the shapes for both signs of the amplitude $a_{2}$ are identical apart from a rotation about $\pi / 2$. Consequently, for vesicles in two dimensions there is no analog to the difference between oblate and prolate ellipsoids in $D=3$. For $D=3$, the shape transformation from the sphere to the energetically lower ellipsoid is of first order, while the metastable sphere becomes unstable with respect to the energetically higher ellipsoid via a second-order transition of the type discussed here. ${ }^{4,8}$ This is a consequence of the fact that the term $O\left(a_{2}^{3}\right)$ does not vanish identically in $D=3$ at $p=p_{2}$ as it does here for $D=2$.

\section{Noncircular shapes}

For $p>p_{2}$, the solutions of the EL equations have to be found numerically. For fixed $p$, the free initial values $u(0)$ and $\gamma(0)$ are varied in order to fulfill $(2.10 \mathrm{~b})$ and $(2.10 \mathrm{~d})$. Let me first focus on the solutions that evolve from the $k=2$ instability. In Fig. 2, solutions of this branch for different values of $p$ are shown and in Fig. 3 characteristic quantities of these solutions are displayed. For $p=p_{3} \simeq 3.34$ the curvature vanishes at the top and bottom of the vesicle [i.e., $u(0)=u(\pi)=0$ ] and for $p>p_{3}$, the shape becomes concave at the top and bottom. In this regime, these $2 \mathrm{D}$ shapes resemble the contour of a discocyte shape of red blood cells. At $p \equiv p_{4} \simeq 5.4$, the shape starts to self-intersect. For even larger pressure one has to add a repulsive interaction term in order to prevent the self-intersection. I do not consider such an extended model here, but restrict the pressure to $p<p_{4}$.

It would again be useful to perform a stability analysis of these noncircular shapes, which represent the $k=2$ branch. This can, in principle, only be determined by a calculation of the second variation $\delta^{2} F$. Since there is no analytic expression available for these shapes, such a 


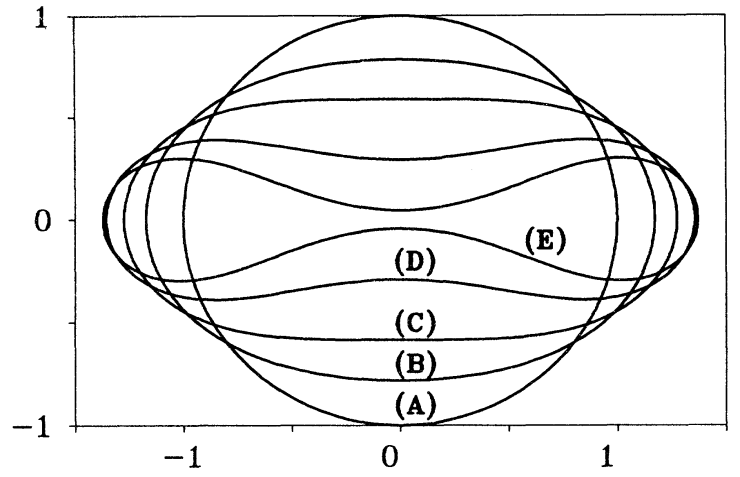

FIG. 2. Free vesicle shapes of the $k=2$ branch for different values of the applied pressure difference p. $p=p_{2}=3(A)$; $p=3.1(B) ; p=p_{3} \simeq 3.34(C) ; p=4.0(D) ; p=5.0(E)$.

computation could only be performed numerically. This has not been attempted so far. Nevertheless, one can expect that these shapes are indeed stable, since they bifurcate from the circle, which is stable with respect to all other harmonic deformations for $p \simeq p_{2}$. By continuity, this stability extends to a finite $p$ interval above $p_{2}$.

In $D=3$, the $k=2$ branch shows an additional instability with respect to the up-down symmetry. This leads finally to stomatocytelike shapes. ${ }^{3}$ Such shapes have also been found in the simulations ${ }^{20,21}$ for $D=2$ at relatively large pressure above $p_{4}$, where the repulsive interaction is relevant. Therefore the model (2.4) without such a repulsion does not produce these shapes.

For $p>3$, the circular shape is a local maximum of the free energy (2.4). Consequently, the instability of the circular shape with respect to the higher harmonics $k \geq 3$ at $p_{c}(k)=\left(k^{2}-1\right)$ cannot be reached from a locally stable shape. Nevertheless, shapes that approach the circle for $p \rightarrow p_{c}(k)+$ and correspond to the $k \geq 3$ branches are

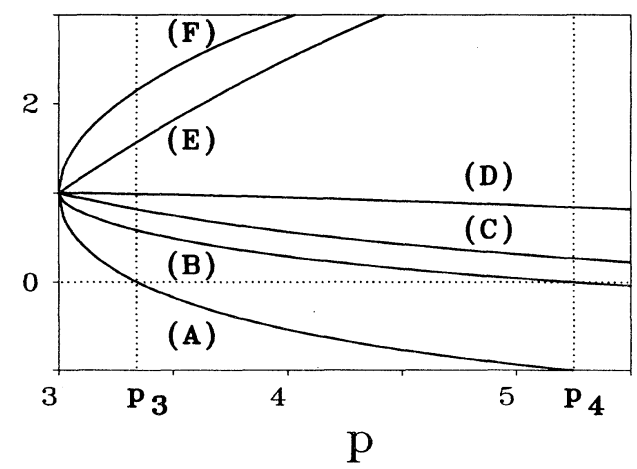

FIG. 3. Characteristic quantities of the solutions of the $k=2$ branch as a function of the pressure $p$ normalized to the corresponding value of the circular shape. Area is $A / \pi(C)$; bending energy, $F_{\kappa} / \pi(E)$; enthalpy, $\left(F_{\kappa}+F_{p}\right) /[\pi(1+p)](D)$; smallest diameter, $[z(0)-z(\pi)] / 2(B)$; minimal and maximal curvature $u^{-}$and $u^{+}$, Eq. (2.20), $(A)$ and $(F)$, respectively. found numerically. These solutions inherit the instability of the circle with respect to a $k=2$ mode and thus are saddle points of the energy. In this paper I focus on the stable shapes which arise from the $k=2$ instability of the circle.

Summarizing, the phase diagram in the $P$ ensemble is rather simple. For $p<p_{2}$, the circular shape is stable, for $p_{2}<p<p_{4}$, the solutions of the $k=2$ branch have lowest energy. Shapes of this branch are elliptical and become continuously of a more discocyte type with increasing pressure. Eventually, the discocyte shapes self-intersect, which defines the limit of applicability of the simple model (2.4)

The $A$ ensemble may be discussed similarly. For given $\epsilon \ll 1$ in Eq. (2.3), one finds, for the amplitude $a_{k}$,

$$
a_{k} \approx \pm\left[2 \epsilon /\left(k^{2}-1\right)\right]^{1 / 2},
$$

setting all other amplitudes equal to 0 . The bending energy follows from Eq. (2.15) as

$$
F_{\kappa} \approx(\kappa / R) \pi\left[1+\left(k^{2}-1\right) \epsilon\right] .
$$

Consequently, the $k=2$ branch gives also the shape with the lowest bending energy for $\epsilon<<1$. In analogy to $D=3$, one can expect that the solutions corresponding to the $k \geq 3$ branches are unstable with respect to the $k=2$ mode. ${ }^{24}$ The results shown in Fig. 3 can also be used to obtain the dependences of characteristic quantities as a function of $\epsilon$. Note for later purposes that the extremal values $u^{ \pm}$of the curvature of the elliptic shapes which are acquired for $s=0$ and $\pi / 2$ are given in lowest order $\epsilon$ by

$$
u^{ \pm} \approx 1 \pm \sqrt{6 \epsilon}
$$

\section{ADHESION IN A CONTACT POTENTIAL}

\section{A. Shape equations and boundary conditions}

In this section, adhesion of a vesicle to a "wall" will be studied. In two dimensions, a wall is, of course, just a line. For a contact potential, the adhesion energy is proportional to the length of the adhering part of the vesicle. Therefore a third contribution $F_{W}$,

$$
F_{W}=-W L^{*} \text {, }
$$

is added to the free energy (2.4). Here $L^{*}$ denotes the length of the adhering part of the vesicle and $W$ is a contact energy per unit length. After rescaling, the total energy $F^{\text {ad }}$ of an adhering vesicle

$$
F^{\mathrm{ad}} \equiv F+F_{W}
$$

may be written as

$$
\begin{aligned}
& F^{\mathrm{ad}}=(2 \kappa / R) \iint_{0}^{s^{*}} d s\left[\frac{1}{2} \dot{\psi}^{2}+p r \sin \psi+\gamma(\dot{r}-\cos \psi)\right] \\
& \left.-w r^{*}\right]
\end{aligned}
$$

where 


$$
\begin{aligned}
& w=W R^{2} / \kappa, \\
& r^{*}=L^{*} /(2 R), \\
& s^{*}=\pi-r^{*} .
\end{aligned}
$$

The EL equations (2.9) remain valid even for the bound vesicle, since the adhesion term $F_{W}$ enters only in the boundary conditions at $s=s^{*}$.

(i) The contact angle obeys $\psi\left(s^{*}\right) \equiv \pi$, since any other angle would imply an infinite curvature energy.

(ii) Variation of the point of contact $s^{*}$, as shown in detail in the Appendix, leads to the nontrivial boundary condition

$$
c\left(s^{*}\right) \equiv \dot{\psi}\left(s^{*}\right)=\sqrt{2 w}
$$

for the contact curvature. For the vesicle with contour length $L=2 \pi R$, rescaling yields

$$
C\left(S^{*}\right)=C\left(s^{*} R\right)=(1 / R) c\left(s^{*}\right)=\sqrt{2 W / \kappa} .
$$

This is indeed the same boundary condition as for the adhesion of vesicles in three dimensions. ${ }^{13}$ It results from the balance between adhesion and curvature energy. This condition is independent of the size of the vesicle and the applied pressure or chosen area. Thus it holds in both ensembles.
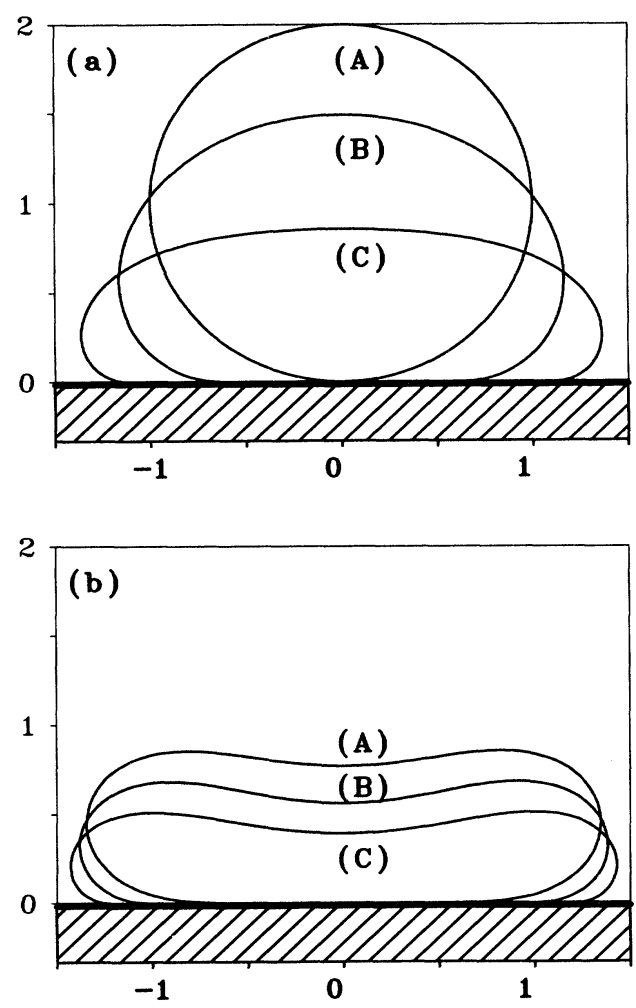

FIG. 4. Adhering shapes for different pressure difference $p$ and increasing contact potential $w$. (a) $p=0$ and $w=0.5(A)$; $w=2.0(B) ; w=5.0(C)$. (b) $p=4.0$ and $w=0.0(A) ; w=2.0(B)$ $w=5.0(C)$. Note that the shape for $p=4.0$ and $w=0.0$ is metastable.
Shapes of adhering vesicles are now found numerical$1 y^{25}$ by solving the EL equations (2.9) with the boundary conditions

$$
\begin{aligned}
& \psi(0)=0, \\
& r(0)=0,
\end{aligned}
$$

and

$$
\begin{aligned}
& \psi\left(s^{*}\right)=\pi \\
& u\left(s^{*}\right)=\sqrt{2 w}, \\
& r^{*} \equiv r\left(s^{*}\right)=\pi-s^{*}
\end{aligned}
$$

The initial values $u(0)$ and $\gamma(0)$ as well as the integration interval $s^{*}$ have to be varied for fixed $p$ and $w$ in order to fulfill Eqs. (3.8). This procedure leads typically to several solutions. Some of them are shown in Fig. 4. The phase diagram is determined by the solution of lowest energy including the free solution at the same conditions. The result depends on the ensemble.

\section{B. Adhesion transition in the $\boldsymbol{P}$ ensemble}

Here the relevant energy is $F_{\kappa}+F_{P}+F_{W}$, with $F_{W}=0$ for the free solution. The resulting phase diagram is shown in Fig. 5. Depending on the pressure, one finds different regimes which can be characterized as follows.

(i) For $p<p_{1} \simeq 1.39$ and $w>w_{a}=\frac{1}{2}$, I find solutions that approach a circular shape as $w$ approaches $w_{a}$ from above [see Fig. 4(a)]. For these solutions, the length of contact $r^{*}$ vanishes continuously as the critical value $w_{a}(p)=\frac{1}{2}$ is approached:

$$
r^{*} \sim\left(w-w_{a}\right)
$$

The energy difference $\Delta F$ between these adhering solutions and the free circle behaves like

$$
\Delta F \sim-\left(w-w_{a}\right)^{2} .
$$

Consequently, the curve $w_{a}(p)=\frac{1}{2}$ denotes a line of con-

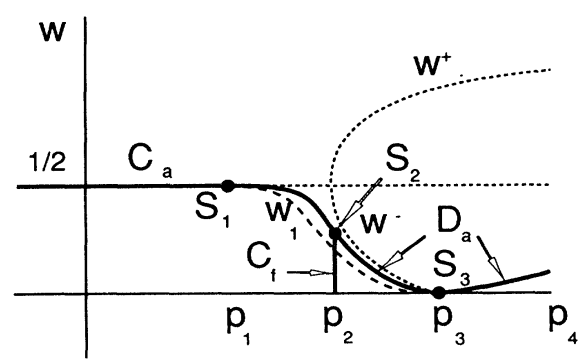

FIG. 5. Phase diagram in the $P$ ensemble. Above the phase boundary $\left(C_{a}, S_{1}, D_{a}, S_{3}, D_{a}\right)$, which defines the curve $w_{a}(p)$, the vesicle is bound; below it, it is free. $C_{a}$ and $D_{a}$ denote a continuous and discontinuous adhesion transition, respectively. $C_{f}$ denotes the continuous transition between the free circular and the free elliptical shapes. The curves $w^{+}, w^{-}$, and $w_{1}$ denote limits that are approached by metastable or unstable solutions (see text for details). 
tinuous adhesion transitions $\left(C_{a}\right)$. Note that for $w=\frac{1}{2}$ the circle indeed fulfills the boundary condition (3.8) for $r^{*}=0$ and thus the circle is a solution to the variation problem with a contact length $r^{*}=0$.

The numerical value of $p_{1}$ follows from a stability analysis of this circle adhering in one point. This solution becomes unstable with respect to a larger contact length at a critical pressure $p_{c, 1} \equiv p_{1}$, which is the smallest positive solution of the implicit equation ${ }^{26}$

$$
\tan \left(\pi \sqrt{1+p_{c}}\right)=-\pi p_{c} \sqrt{1+p_{c}} .
$$

(ii) For $p_{1}<p \leq p_{2}=3$, one finds two solutions with $r^{*}>0$ for $w_{1}(p)<w<\frac{1}{2}$. One of them has a lower energy than the circle for $w_{a}(p)<w<\frac{1}{2}$ with $w_{1}(p)<w_{a}(p)$. The other has a higher energy than the circle and approaches the circle for $w \rightarrow \frac{1}{2}-$. Both solutions merge for $w \rightarrow w_{1}(p)+$ and disappear for lower values of $w$. Thus the phase boundary between free and adhering shapes for this $p$ range is given by a line of first-order transitions $\left(D_{a}\right)$ at $w_{a}(p)$.

(iii) The curves $\left(C_{a}\right)$ and $\left(D_{a}\right)$ meet at the tricritical point $\left(S_{1}\right)$ with $p=p_{1}$ and $w=\frac{1}{2}$. In vicinity of $\left(S_{1}\right)$, the phenomenology of the adhesion transitions described so far may be understood in terms of a Landau-type theory for the "order" parameter $r^{*}$. The energy difference $\Delta F$ between an adhering and the free circular solution reads

$$
\Delta F=-d_{1} \Delta w r^{*}-d_{2} \Delta p r^{* 2}+d_{3} r^{* 3},
$$

with

$$
\begin{aligned}
& \Delta w \equiv w-\frac{1}{2}, \\
& \Delta p \equiv p-p_{1} .
\end{aligned}
$$

Here $d_{1,2,3}$ are smooth functions of $\Delta w$ and $\Delta p$ which assume nonvanishing positive values at $\Delta w=\Delta p=0$. Extremizing $\Delta F$ leads to one local minimum for $\Delta p<0$ and $\Delta w>0$ and the power laws (3.9) and (3.10). For $\Delta p>0$ and $\Delta w<0, \Delta F$ has two extrema with $r^{*}>0$ which correspond to the solutions found numerically in this regime. One of them is a local maximum and thus corresponds to an unstable shape. For $\Delta p>0$, the phase boundary $\left(D_{a}\right)$ follows from (3.12) as

$$
w_{a}(p)-\frac{1}{2} \sim-\Delta p^{2} .
$$

(iv) For $p=p_{2}$, the free vesicle undergoes the secondorder transition $\left(C_{f}\right)$ from the circular to an elliptical shape. Consequently, the point $\left(S_{2}\right)$ at $p=p_{2}$ and $w=w_{a}\left(p_{2}\right)$ is a critical end point, where this free (critical) shape coexists with an (uncritical) bound shape.

(v) For $p_{2} \leq p \leq p_{3} \simeq 3.43$, the phase boundary $w_{a}(p)$ and the curve $w_{1}(p)$ decrease continuously with $p$ and merge at $w=0$ and $p=p_{3}$. These values define a higherorder critical point $\left(S_{3}\right)$. For $p=p_{3}$, the adhesion transition is again of second order with

$$
r^{*} \sim w^{1 / 4}, \quad \Delta F \sim w^{5 / 4} .
$$

This higher-order critical point is indeed an azeotropic point for the following reason. (vi) For $p>p_{3}$ the phase boundary $w_{a}(p)$ between free and adhering solutions increases and this transition $\left(D_{a}\right)$ is of first-order again. One finds in the range $0<w<w_{a}(p)$ adhering shapes which have a higher energy than the free discocytelike shapes which are thus only metastable. In Fig. 4(b) bound shapes for $p>p_{3}$ are shown. For even larger pressure bound solutions also self-intersect.

Summarizing, the shapes of lowest energy are bound for $w>w_{a}(p)$ and free for $w<w_{a}(p)$. The transition between both is either of second order (for $p<p_{1}$ and $p=p_{3}$ ) or first order.

Let me briefly comment on additional bound solutions of the EL equations and the boundary conditions, which correspond to locally unstable states. I already mentioned a second bound solution found for $p_{1}<p<p_{2}$ between $w_{1}(p)<w<\frac{1}{2}$. Since this solution approaches the circle for $w \rightarrow \frac{1}{2}-$, it is influenced by the instability of this circle at $p=p_{2}$. Indeed, one finds for $p>p_{2}$ a bound solution for $w_{1}(p)<w<w^{-}(p)$ approaching the free elliptical shape with the long axis parallel to the wall for $w \rightarrow w^{-}(p)-$. The numerical value of $w^{-}(p)$ is given by $w^{-}=\left(u^{-}\right)^{2} / 2$, where $u^{-}$is the minimal curvature of the elliptical shape [compare Fig. 3 and Eq. (2.20)]. The relation between $w^{-}$and $u^{-}$follows from the fact that, for this value of the contact potential, the free elliptical solution adhering in one point is indeed a solution of the EL equations and the boundary conditions (3.8) as it is the circle for $w=\frac{1}{2}$. For $w^{-}(p)<w<\frac{1}{2}$, one finds numerically solutions with $r^{*}<0$, which are unphysical. For $\frac{1}{2}<w<w^{+}(p)$, with $w^{+}(p)=\left(u^{+}\right)^{2} / 2$, these shapes, however, possess $r^{*}>0$ and approach the free elliptic shape adhering in one point with the small axis parallel to the wall for $w \rightarrow w^{+}(p)-$.

A further class of unstable bound solutions bifurcates from the circle at $p=p_{c, n}$, with $n \geq 2$, which correspond to larger solutions of Eq. (3.11). These bound solutions are related to the free $k \geq 3$ branches. Since $p_{c, n}>p_{2}$ for $n \geq 2$, these bound solutions inherit the instability with respect to the $k=2$ mode from the circle and thus correspond to locally unstable solutions.

\section{Adhesion transition in the $\boldsymbol{A}$ ensemble}

The solutions discussed in Sec. II B as a function of $p$ also correspond to solutions as a function of $\epsilon$. Thus no additional shapes occur in the $A$ ensemble. The energy, however, which determines the phase diagram, is now given by $F_{\kappa}+F_{W}$ without the enthalpy term. The phase diagram is shown in Fig. 6 .

For $0 \leq \epsilon \leq \epsilon_{1}$, with $\epsilon_{1} \simeq 0.18$, the state of lowest energy is bound for $w>w_{a}(\epsilon)=w^{-}(\epsilon)=\left(u^{-}\right)^{2} / 2$. Note that $w^{-}(\epsilon)$ is identical to $w^{-}(p)$, as discussed in Sec. II B, but now considered as a function of $\epsilon$. From Eq. (2.20), one finds the asymptotic behavior $w_{a}(\epsilon) \approx \frac{1}{2}-\sqrt{6 \epsilon}$. For $w \rightarrow w_{a}(\epsilon)+$, the contact length $r^{*}$ and the energy difference $\Delta F$ obey

$$
\begin{aligned}
& r^{*} \sim\left[w-w_{a}(\epsilon)\right], \\
& \Delta F \sim-\left[w-w_{a}(\epsilon)\right]^{2} .
\end{aligned}
$$




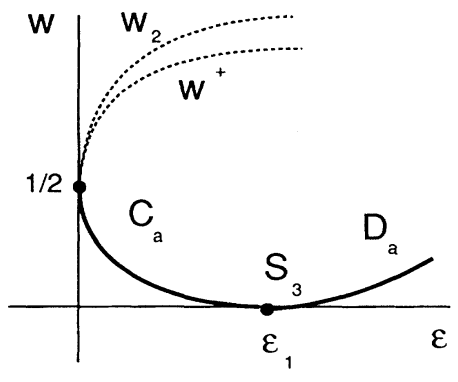

FIG. 6. Phase diagram in the $A$ ensemble. Above the phase boundary $\left(C_{a}, S_{3}, D_{a}\right)$, which defines the curve $w_{a}(\epsilon)$, the vesicle is bound; below it, it is free. The curves $w^{+}$and $w_{2}$ denote limits that are approached by metastable or unstable solutions (see text for details).

Thus the adhesion transition is continuous $\left(C_{a}\right)$ in this range. Apart from this bound solution, I find in the range $w^{+}(\epsilon)<w<w_{2}(\epsilon)$, with $w^{+}(\epsilon) \equiv\left(u^{+}\right)^{2} / 2$, two additional bound solutions with higher energy. The energetically lower of them approaches the free ellipse for $w \rightarrow w^{+}(\epsilon)+0$, with the small semiaxis of the ellipse parallel to the wall. This solution is a local minimum and thus corresponds to a metastable bound state. The energetically higher bound solution corresponds to a maximum and is unstable. Both merge at $w=w_{2}(\epsilon)$ and disappear for $w>w_{2}(\epsilon)$.

For $\epsilon=\epsilon_{1}$, the free elliptical solution has a vanishing curvature $u^{-}$, and solves the boundary condition (3.8) for $w=0$ with $r^{*}=0$. This solution is approached for $w \rightarrow 0$ by bound states which have a lower energy than the free state. This point $\left(S_{3}\right)$ corresponds to the azeotropic point in the $P$ ensemble. In the $A$ ensemble, however, it is a tricritical point, since for $\epsilon>\epsilon_{1}$, the phase boundary $w_{a}(\epsilon)$ increases again as $w_{a}(\epsilon) \sim\left(\epsilon-\epsilon_{1}\right)^{2}$. It separates bound states with finite $r^{*}$ from the free discocytetype states and denotes a discontinuous transition $\left(D_{a}\right)$. With increasing $\epsilon$ self-intersecting states are reached.

Note that analogs to the curves $w^{+}$and $w^{-}$can also be derived for the free shapes corresponding to the $k \geq 3$ branches, which are also approached by additional bound states. These states have, however, a higher energy than those derived from the $k=2$ branch discussed here and, moreover, are presumably unstable with respect to the $k=2$ mode.

\section{Comparison with the adhesion transition in $D=3$}

The phase diagrams in Fig. 5 for the $P$ ensemble and in Fig. 6 for the $A$ ensemble can be compared with the corresponding ones for $D=3 .^{13,14}$ of course, the $A$ ensemble discussed here for $D=2$ must be compared with the $V$ ensemble for $D=3$, where the volume is kept constant. As a basic result, any of the transitions discussed here are also found for $D=3$. The main difference, however, is that for $D=3$ additional transitions occur and some of the transitions discussed here for $D=2$ are transitions between metastable states in $D=3$. One reason for this effect is again the difference between prolate and oblate ellipsoids for $D=3$.

Consider, e.g., the continuous transition $\left(C_{a}\right)$ in the $A$ ensemble and $\epsilon<\epsilon_{1}$. For $D=2$, the bound shape approaches an elliptical shape for $w \rightarrow w_{a}(\epsilon)+$. The analogous situation for $D=3$ is the approach to a free oblate ellipsoid with decreasing strength $w$. If, however, the prolate ellipsoid is energetically lower than the oblate one, a first-order adhesion transition between a bound oblate shape with small but finite area of contact and the unbound prolate ellipsoid at the same volume must be encountered. The continuous transition between the bound oblate shape and the free oblate is then a transition between metastable states. This is precisely the case in $D=3$ for zero spontaneous curvature. ${ }^{14}$ The second reason for the additional transitions for $D=3$ is the existence of stomatocytelike shapes, which do not occur in the model (2.4) as discussed in Sec. II C.

A more technical difference is the occurrence of logarithmic factors in power laws such as Eq. (3.16) for $D=3$ which are absent here. They arise from singularities of the shape equations at the axis of symmetry for $D=3$.

\section{ADHESION IN A FINITE-RANGE POTENTIAL}

The description given in Sec. III may also be considered as the limit where the range of the potential $Z_{0}$ is small compared to the vesicle size $R$. For a long-ranged potential the whole vesicle may be exposed to the influence of the wall. It is of special interest to discuss the crossover from a long-ranged to a short-ranged adhesion potential. I address this question in this section.

Instead of the contact potential $F_{W}$, now the potential term $F_{V}$ with

$$
F_{V}=\oint d S V(Z(S))
$$

is added to the free energy (2.4). $Z(S)$ is the local distance of the vesicle of contour length $L=2 \pi R$ from the wall at $Z=0$. After rescaling the contour length, one finds

$$
F_{V}=(\kappa / R) \oint d s v(z(s))
$$

with $z \equiv Z / R$ and the rescaled potential

$$
v(z) \equiv\left(R^{2} / \kappa\right) V(z R)
$$

The total energy $F^{\mathrm{ad}}=F_{\kappa}+F_{P}+F_{V}$ can now be written in the form

$$
\begin{aligned}
& F^{\mathrm{ad}}=(2 \kappa / R) \int_{0}^{\pi} d s\left[\frac{1}{2} \dot{\psi}^{2}+p z \cos \psi+\gamma(\dot{r}-\cos \psi)\right. \\
& +\delta(\dot{z}+\sin \psi)+v(z)] \text {. }
\end{aligned}
$$

Note that I used a different but equivalent form the enthalpy term and that a second Lagrange multiplier $\delta(s)$ is introduced since the coordinate $z$ shows up explicitly. The complete set of the EL equations now reads 


$$
\begin{aligned}
& \dot{\psi}=u, \\
& \dot{u}=-p z \sin \psi+\gamma \sin \psi+\delta \cos \psi, \\
& \dot{\gamma}=0 \\
& \dot{\delta}=d v / d z+p \cos \psi, \\
& \dot{r}=\cos \psi \\
& \dot{z}=-\sin \psi
\end{aligned}
$$

The boundary condition for $\psi$ and $r$ are

$$
\psi(0)=\psi(\pi)=r(0)=r(\pi)=0 .
$$

Since $z(0)$ and $z(\pi)$ are arbitrary, these boundary values must be varied too, which leads to the boundary conditions for the Lagrange multiplier $\delta$ :

$$
\delta(0)=\delta(\pi)=0 \text {. }
$$

Note that these boundary conditions automatically guarantee an analytic shape at $s=0$ and $\pi$. The explicit boundary condition (3.5) for the contact curvature that was present in the case of the contact potential has disappeared since there is no point of contact for a smooth potential $v(z)$. I anticipate, however, that this boundary condition is recovered in the limit when the range of the potential goes to zero.

For any given potential $v(z)$ and pressure $p$, one now has to solve the boundary value problem for $\psi, r$, and $\delta$ with the adjustable initial values $u(0), z(0)$, and $\gamma$. As a concrete example, I consider the following two-parameter potentials:

$$
v(z)=v^{0}\left[\left(\frac{z_{0}}{z}\right)^{4}-2\left(\frac{z_{0}}{z}\right)^{2}\right] .
$$

This potential has a minimum for $z=z_{0}$ with a depth $v\left(z_{0}\right)=-v^{0}$, which read $Z_{0} \equiv z_{0} R$ and $V^{0} \equiv\left(\kappa / R^{2}\right) v^{0}$ in the unscaled quantities, respectively. Any of the results given further below will also hold for a general smooth potential which vanishes for $Z \rightarrow \infty$ and can be characterized by a depth $V^{0}$ and a typical length scale of range $Z_{0}$.

In Fig. 7, vesicle states bound in such a potential are shown. These illustrate the general feature which can be explained for both ensembles simultaneously. Two regimes must be distinguished.

(i) For $Z_{0}<<R$, which defines the short-ranged case, only the adjacent part of the vesicle is directly influenced by the potential and the results can be compared with those for a contact potential. First, consider $v^{0}>w_{a}\left({ }_{\epsilon}^{p}\right)$ fixed. [The notation $(\underset{\epsilon}{p})$ denotes the dependence on $p$ or $\epsilon$ in either ensemble.] Then the vesicle indeed approaches, for $z_{0} \rightarrow 0$, the same shape as in the presence of a contact potential with $w$ given by $v^{0}$ [compare Fig. 7(a)]. ${ }^{27}$ Thus the boundary condition (3.5) is recovered as a result of the solution. If $v^{0}<w_{a}\left({ }_{\epsilon}^{p}\right)$, however, the shape approaches, for $z_{0} \rightarrow 0$, the free shape at the same $p$ or $\epsilon$, but the vesicle remains pinned by the narrow potential well to the wall and in this sense it is not unbound [compare Fig. $7(\mathrm{~b})$ ]. Multiplying the length of the contour exposed to
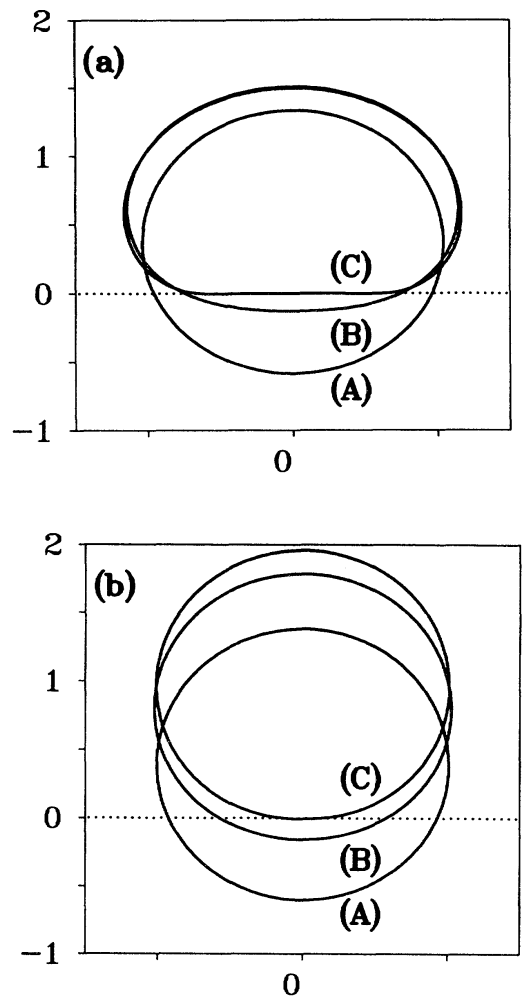

FIG. 7. Vesicle shapes in the finite-range potential $v(z)$, Eq. (4.8), for $p=0$. The dotted line shows the minimum of the potential, i.e., the vertical axis denotes $z-z_{0}$. (a) $v^{0}=2.0$ and $z_{0}=5.0(A) ; z_{0}=1.0(B) ; z_{0}=0.03(C)$. The shape $(C)$ should be compared with the shape $(B)$ in Fig. 4(a). (b) Same as (a), but for $v^{0}=0.4$. Shape $(C)$ is almost circular, but pinned by the potential minimum.

the potential with the potential depth, one finds that such a pinned state gains an energy difference

$$
|\Delta F| \sim V^{0} \sqrt{Z_{0} R} \text { for } V^{0}<V_{a}^{0} \equiv w_{a} \kappa / R^{2}
$$

compared to the unbound free shapes. Although this energy vanishes for $Z_{0} \rightarrow 0$, the state of lowest energy in a potential with finite range is thus always bound. Consequently, the role of the adhesion transitions $\left(C_{a}\right)$ and $\left(D_{a}\right)$ found for a contact potential can be extended for a finite-range potential as follows (Fig. 8).

The discontinuous transition $\left(D_{a}\right)$ extends to a whole sheet $v_{a}^{0}\left(z_{0},{ }_{\epsilon}^{p}\right)$, with $v_{a}^{0}\left(0,{ }_{\epsilon}^{p}\right)=w_{a}(\underset{\epsilon}{p})$. It denotes the shape transition between two different bound states. One of them corresponds to the bound state found for the contact potential case, while the other is the pinned state which approaches the free shape for $z_{0} \rightarrow 0$. As for a contact potential, this transition is governed by the competition between bending and adhesion energy. This sheet of first-order transitions terminates in a curve $v_{a}^{0}\left[z_{0, c}(\underset{\epsilon}{p})\right]$ of second-order transitions which is the continuation of the $\left(C_{a}\right)$ transition (compare Fig. 8). Thus, for $z_{0}>z_{0, c}$ (with $z_{0, c}=0$ if $\epsilon<\epsilon_{1}$ or $p<p_{1}$, respectively), there is only one minimum shape in the potential. In this case the distinc- 


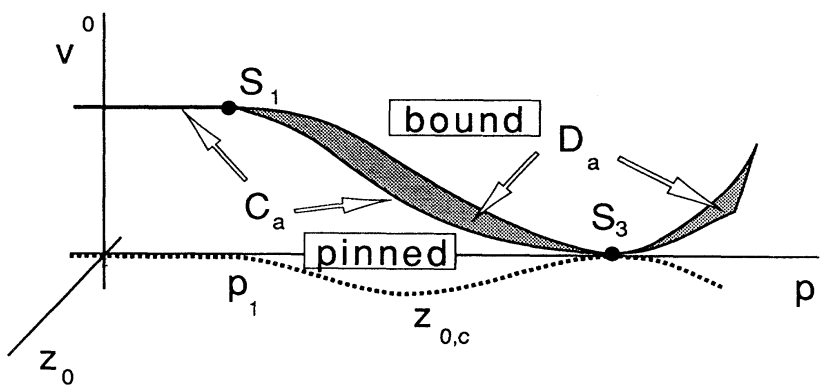

FIG. 8. Schematic phase diagram for the $P$ ensemble and finite $z_{0}$. The sheets $\left(D_{a}\right)$ given by $v_{a}^{0}\left(z_{0}, \frac{p}{\epsilon}\right)$ denote first-order transitions between a bound state with finite contact length and a pinned state with the free shape. These first-order transitions terminate in a curve $\left(C_{a}\right)$ of second-order transitions. The dashed line denotes the projection of this curve onto the $v^{0}=0$ plane. For $z_{0}>z_{0, c}$ the distinction between the pinned and bound states no longer holds. An analogous phase diagram holds in the $A$ ensemble.

tion between bound and pinned states no longer applies and the vesicle approaches continuously its free shape with decreasing depth of the potential.

(ii) For $R \lesssim Z_{0}$, which defines the long-ranged case, the whole bound vesicle is exposed to the adhesion potential $F_{V}$, which leads, in the $P$ ensemble, to a deviation from the circular shape at any $p$. Thus the transition $\left(C_{f}\right)$ for free vesicles has been smeared out. The deviations from the free shape at the same $p$ are significant if the variation of the potential along the vesicles contour becomes comparable to the energy $F \sim \kappa / R$. This happens at an amplitude $V^{0}=V_{f}^{0}$, which can be estimated by expanding the potential around its minimum as

$$
V_{f}^{0} \sim \kappa Z_{0}^{2} / R^{4} .
$$

Thus, for $V^{0} \ll V_{f}^{0}$, the bound vesicles have more or less their free shape and gain an energy

$$
|\Delta F| \sim V^{0} R
$$

while for $V^{0} \gtrsim V_{f}^{0}$ the adhesion leads to significant deviations from these free shapes.

\section{INFLUENCE OF FLUCTUATIONS}

\section{A. Shape fluctuations of free vesicles}

The analysis given in Sec. II was based on a study of the EL equations. These calculations give the state of lowest energy and thus correspond formally to temperature $T=0$. At any finite temperature, fluctuations have to be considered. The relevant criterion for the influence of fluctuations on free vesicles is basically given by the relation between the linear size $R$ of the vesicle and the persistence length $L_{\kappa}$ [Eq. (1.1)]. For $R \gg L_{\kappa}$, fluctuations are strong and the vesicle must be described as a closed self-avoiding walk. This regime has been studied by scaling theories and Monte Carlo simulations. ${ }^{20-22}$ For
$R<<L_{\kappa}$, however, the influence of fluctuations is moderate and can be studied perturbatively.

First, I consider the $P$ ensemble. For $p<p_{1}$, the shape of lowest energy for a free vesicle is a circle. Fluctuations around this shape have already been introduced in Eq. (2.11) for the stability analysis of Sec. II. Equation (2.16) yields, for the mean amplitudes in the Gaussian approximation,

$\left\langle a_{k}^{2}\right\rangle=\left\langle b_{k}^{2}\right\rangle=\frac{R}{\pi L_{\kappa}\left(k^{2}-1\right)\left(k^{2}-1-p\right)} \quad$ with $k \geq 2$.

These fluctuations are small for $R \ll L_{\kappa}$, provided $p \ll p_{2}=3$. Various characteristic quantities can now be computed to order $R / L_{\kappa}$ such as, e.g., the mean radius $\langle\bar{\rho}\rangle$ of the vesicle; compare Eqs. (2.11) and (2.13):

$$
\langle\bar{\rho}\rangle=R\left(1+\left\langle a_{0}\right\rangle\right) \approx R\left(1-\alpha R / L_{\kappa}\right) .
$$

Here, $\alpha$, with

$$
\alpha=\frac{1}{2 \pi} \sum_{k(\geq 2)} \frac{k^{2}}{\left(k^{2}-1\right)\left(k^{2}-1-p\right)},
$$

measures the fraction of length that is absorbed in the fluctuations. Of course, at any finite $T$, a particular shape with frozen fluctuations is noncircular. A possible measure of this effect is the mean aspherity $S$, which is defined $^{21}$ by $S=\left\langle R_{G_{1}}^{2}\right\rangle /\left\langle R_{G_{2}}^{2}\right\rangle$, where $R_{G_{1,2}}$ are the larger and smaller eigenvalues of the tensor of inertia for the vesicle. One finds $S-1 \sim R /\left[L_{\kappa}\left(p_{2}-p\right)\right]$. The sharp transition $\left(C_{f}\right)$, which separates circular from noncircular shapes for $T=0$, has thus disappeared. For $p \simeq p_{2}$, even the Gaussian approximation breaks down and higher order terms in Eq. (2.16) become relevant. For $p_{2}<<p<p_{4}$, however, the computation of Gaussian fluctuations about the noncircular shape should be possible. One expects that the amplitude $a$ of the mode, which corresponds to the lowest shape deformation, also behaves like $\left\langle a^{2}\right\rangle \sim R / L_{\kappa}$.

In the $A$ ensemble, the problem is in principle even more subtle, since the fluctuations have to respect the two constraints of constant contour length and constant area. Since no transitions between different shapes are found in the analysis of the EL equations, I do not except that fluctuations will modify the mean shapes significantly provided $R<<L_{\kappa}$.

\section{B. Fluctuations of vesicles in a finite-range potential}

For a vesicle bound by a contact potential, a perturbative discussion of shape fluctuations especially of the bound part of the contour is not obvious. One may, however, consider the strength $W$ of the contact potential as an effective value which is already renormalized by fluctuations. In the following, I discuss fluctuations for vesicles bound in a potential with finite range. As above, only in the case $R<L_{\kappa}$ is considered.

For $T \neq 0$ the notion of a bound vesicle is only meaningful if the typical fluctuation of the vesicle's position in 
the direction perpendicular to the wall is smaller than the range of the potential $Z_{0}$. For larger fluctuations the vesicle will unbind. Of course, there is no sharp transition between the characterization "bound" and "free," since the vesicle as a finite system will overcome the barrier $|\Delta F|$, which is the energy difference between a bound and a free state, in a finite time of the order of $e^{|\Delta F| / T}$.

Let me first comment on the case $|\Delta F| \gg T$, for which the bound state is sufficiently long lived. Then shape fluctuations of this state can be computed in principle within the Gaussian approximation by diagonalizing the second variation of $F^{\text {ad }}$ in Eq. (4.4). Although this can only be performed numerically and has not been attempted so far, one expects again for a typical amplitude $a,\left\langle a^{2}\right\rangle \sim T$. At the second-order transition $C_{a}$ for $v^{0}=v_{a}^{0}\left(z_{0, c}\right)$ (compare Fig. 8), however, one of the modes becomes again a soft mode which cannot be treated within the Gaussian approximation.

For $|\Delta F| \lesssim T$, the fluctuations will include one mode that is related to the fluctuation of the position of the vesicle perpendicular to the wall. An estimate for the potential depth $V_{u}^{0}$ for which that mode becomes dominant and thus the unbinding occurs can be obtained easily if one takes the criterion that $|\Delta F| \simeq T$ for $V^{0}=V_{u}^{0}$. From Eq. (4.9) and (4.11), one finds

$$
V_{u}^{0} \simeq\left\{\begin{array}{l}
(T / R) \text { for } R \lesssim Z_{0} \\
T / \sqrt{R Z_{0}} \text { for } Z_{0}<R \lesssim R_{c} .
\end{array}\right.
$$

The upper limit $R_{c}$, with

$$
R_{c} \equiv L_{k}^{2 / 3} Z_{0}^{1 / 3} \text {, }
$$

arises from the consistency requirement that $V_{u}^{0}<V_{a}^{0}$, which was assumed when using Eq. (4.9) for $|\Delta F|$. The breakdown of this relation for $R>R_{c}$ indicates that large vesicles will not enter the pinned regime because the energy gain $|\Delta F|$ of such a pinned state would be smaller than the thermal energy $T$. Therefore these large vesicles unbind at values of the potential depth $V^{0}$ for which the $T=0$ analysis predicts bound vesicles with a finite contact length. This regime $R>R_{c}$ can also be attacked from a different point of view as follows.

Consider an open semiflexible polymer of length $R$ bound to a wall by a potential which decays faster than $\sim Z^{-2 / 3}$. For $R \rightarrow \infty$ (with $R \ll L_{\kappa}$ implicitly assumed) such an open "vesicle" will unbind due to its shape fluctuations at a finite potential depth $V_{u \text {,open }}^{0}{ }^{28,29}$ For a square-well potential with a range $Z_{0}$ and depth $V^{0}$, the critical amplitude $V_{u \text {,open }}^{0}$ scales like

$$
V_{u, \text { open }}^{0} \sim \frac{T}{L_{\kappa}^{1 / 3} Z_{0}^{2 / 3}}
$$

Let me assume that for large bound vesicles, with a finite fraction of their contour bound in the potential well, the constraint of a fixed contour length becomes irrelevant for the unbinding. Then the critical amplitude $V_{u \text {,open }}^{0}$ should also give the critical amplitude for the unbinding

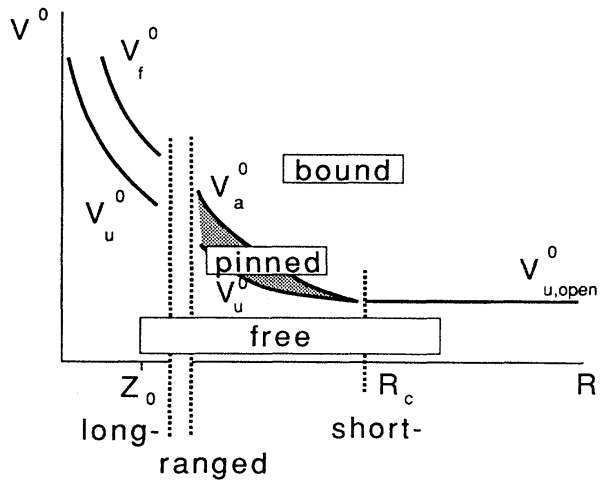

FIG. 9. Schematic phase diagram for the adhesion of vesicles in a finite-range potential including the effect of fluctuations. For potential depth $V^{0}>V_{f}^{0}, V_{a}^{0}, V_{u \text {,open }}^{0}$ the vesicles are bound. In the long-ranged case, they are deformed by the potential, while in the short-ranged case they have a finite contact length. In the shaded region, they are pinned, but have their free shape. The transition between bound and pinned states exists only if, for a contact potential at the same pressure or area, the adhesion transition is of first order. For $R<R_{c}$, vesicles unbind via fluctuations of their position at $V_{u}^{0}$, while for $R>R_{c}$, they unbind via shape fluctuations at $V_{u \text {,open }}^{0}$.

of closed vesicles provided the vesicle is indeed bound with a finite fraction of its contour to the wall for $T=0$. This latter condition restricts the whole argument to vesicles with such a size that $V_{a}^{0}(R)<V_{u \text {,open. This relation is }}^{0}$ fulfilled for $R>R_{c}$ with the same crossover radius $R_{c}$ of Eq. (5.5) appearing already in Eq. (5.4). If this reasoning captures the essential physical mechanism, the results of the simple estimates given in this section of the influence of fluctuations on the unbinding of vesicles can be summarized in the schematic phase diagram, Fig. 9.

In a long-ranged potential $\left(R \lesssim Z_{0}\right)$, a decreasing depth $V^{0}$ first leads to a smooth crossover at $V^{0} \simeq V_{f}^{0}$ from the deformed to a nearly free shape and finally to unbinding via fluctuations of the vesicles position at $V^{0} \simeq V_{u}^{0}$. In a short-ranged potential $\left(Z_{0}<R\right)$, two different regimes must be distinguished, depending on the size of the vesicle.

(i) Small vesicles, with $R<R_{c}$, may first undergo a transition from a bound state with finite contact length to the pinned state with nearly free shape for $V^{0} \simeq V_{a}^{0}$. This holds if, for a contact potential, the adhesion transition at the same $\epsilon$ or $p$ is first order. Of course, this transition between bound and pinned states will be smeared out because of the finite activation energy between these two states. If the transition for a contact potential is second order, the vesicle approaches continuously its free shape with decreasing potential. In both cases, these small vesicles unbind via fluctuations of their position with further decreasing strength of the potential at $V^{0} \simeq V_{u}^{0}$.

(ii) Large vesicles, with $R>R_{c}$, cannot enter the regime of pinned states since they will already have unbound via shape fluctuations especially of the bound part of their contour at $V^{0} \simeq V_{u \text {,open }}^{0}$. 


\section{CONCLUSION}

The main results derived in this paper on the adhesion of vesicles in two dimensions may be summarized in three points.

(i) A detailed investigation for adhesion in a contact potential was given. As a basic analytic result, one finds an universal boundary condition for the contact curvature. Shapes of bound vesicles have been computed and a first- or second-order adhesion transition was found in both ensembles.

(ii) General features for the adhesion in a smooth potential with finite range were studied illustratively for a two-parameter potential. In such a model, the state of lowest energy is always bound in the absence of fluctuations. The adhesion transition found for a contact potential corresponds in this model to a transition between a bound and a pinned state, where the vesicle acquires its free shape but remains pinned in the potential minimum.

(iii) The influence of fluctuations leads to two different regimes for the unbinding. Small vesicles unbind via fluctuations of their position, while large vesicles unbind via shape fluctuations.

These results could be tested by computer simulations, which have been performed so far only for a linear potential. ${ }^{22}$ For a potential with finite range, it would be especially valuable to investigate the pinned regime and the crossover region $R \simeq R_{c}$, where the energy-driven transition between the bound and pinned states interferes with the unbinding induced by shape fluctuations. Simulations at constant contour length and enclosed area should reveal the influence of these constraints on fluctuations.

An equivalent adhesion transition exists also in three dimensions. ${ }^{13,14}$ The analysis given here for two dimensions reveals already the same basic mechanisms, even though the shape equations are simpler. In contrast to this approach based on the minimization of the bending energy, which can be transferred relatively easily between two and three dimensions, the difference in time needed for reliable computer simulations for both cases is enormous. Therefore it may also be worthwhile to use adhesion in two dimensions as a testing ground for the three-dimensional problem and first to compare theory and computer simulations for this case.

Finally note that the adhesion of long tubular vesicles to a planar substrate in three dimensions ${ }^{30}$ is equivalent to the problem studied here, as long as the tube does not bend its axis. Therefore the results obtained by minimization in Secs. II-IV also hold for this adhesion problem, while the unbinding due to shape fluctuations is quite different since it involves bending modes of the whole tube.

\section{ACKNOWLEDGMENTS}

It is a pleasure to thank R. Lipowsky for many stimulating discussions and a critical reading of the manuscript. This work was supported by the Deutsche Forschungsgemeinschaft through Sonderforschungsbereich No. 266 .

\section{APPENDIX}

I derive the boundary condition for the contact curvature from the solution to the variational problem $\delta F^{\text {ad }}=0$ with $F^{\text {ad }}$ from Eq. (3.3). Generally speaking, this is a variational problem with a variable end point $\left(s^{*}\right)$, an auxiliary condition $(\dot{r}=\cos \psi)$, and a boundary function $\left[-w r\left(s^{*}\right)\right]$. The general solution to such a problem can be found, e.g., in Ref. 31 . I give here a simple version.

Write $F^{\text {ad }}$ (with $2 \kappa / R=1$ without loss of generality) in the form

$$
F^{\mathrm{ad}}=\int_{0}^{s^{*}} f(\psi, \dot{\psi}, r, \dot{r}, \gamma) d s+g\left(r\left(s^{*}\right)\right),
$$

with $f=\frac{1}{2} \dot{\psi}^{2}+p r \sin \psi+\gamma(\dot{r}-\cos \psi)$ and $g=-w r$. Consider the variation

$$
\begin{aligned}
& \psi(s)=\psi_{0}(s)+\epsilon \eta_{\psi}(s), \\
& r(s)=r_{0}(s)+\epsilon \eta_{r}(s),
\end{aligned}
$$

where $\psi_{0}$ and $r_{0}$ denote the extremal solution to be found. (The subscript 0 has been omitted in the main part.) These obey, as $s_{0}^{*}$,

$$
\begin{aligned}
& \psi_{0}\left(s_{0}^{*}\right)=\pi, \\
& r_{0}\left(s_{0}^{*}\right)=\pi-s_{0}^{*} .
\end{aligned}
$$

Likewise, the varied solution has to fulfill

$$
\begin{aligned}
& \psi\left(s^{*}\right)=\pi, \\
& r\left(s^{*}\right)=\pi-s^{*},
\end{aligned}
$$

with an unknown $s^{*}(\epsilon)$, which can be expanded as

$$
s^{*}(\epsilon)=s_{0}^{*}+\left.\epsilon \frac{d s^{*}}{d \epsilon}\right|_{\epsilon=0}+O\left(\epsilon^{2}\right) .
$$

The boundary values in Eqs. (A4) can also be expanded as

$$
\begin{aligned}
\psi\left(s^{*}(\epsilon)\right)= & \psi_{0}\left(s_{0}^{*}\right)+\epsilon\left[\left.\dot{\psi}\left(s_{0}^{*}\right) \frac{d s^{*}}{d \epsilon}\right|_{\epsilon=0}+\eta_{\psi}\left(s_{0}^{*}\right)\right] \\
& +O\left(\epsilon^{2}\right), \\
r\left(s^{*}(\epsilon)\right)= & r_{0}\left(s_{0}^{*}\right)+\epsilon\left[\left.\dot{r}\left(s_{0}^{*}\right) \frac{d s^{*}}{d \epsilon}\right|_{\epsilon=0}+\eta_{r}\left(s_{0}^{*}\right)\right] \\
& +O\left(\epsilon^{2}\right),
\end{aligned}
$$

Comparing Eqs. (A4) and (A6), using Eq. (A3) and the relation $\dot{r}\left(s_{0}^{*}\right)=\cos \psi\left(s_{0}^{*}\right)=-1$, one arrives at

$$
\begin{aligned}
& \eta_{r}\left(s_{0}^{*}\right)=0, \\
& \eta_{\psi}\left(s_{0}^{*}\right)=-\left.\dot{\psi}\left(s_{0}^{*}\right) \frac{d s^{*}}{d \epsilon}\right|_{\epsilon=0} .
\end{aligned}
$$

Equation (A7a) restricts the admissible variations in $r$, while (A7b) gives the variation of the "new" end point $s^{*}(\epsilon)$ as a function of the variation in $\psi$ and the curvature $\dot{\psi}$ at the "old" end point $s_{0}^{*}$.

Now, return to $F^{\text {ad }}$ in Eq. (A1). Inserting Eq. (A2) and the upper boundary $s^{*}$, Eq. (A5), into Eq. (A1), one finds, after a partial integration, the derivative 


$$
\begin{aligned}
\left.\frac{d F^{\mathrm{ad}}}{d \epsilon}\right|_{\epsilon=0}= & \int_{0}^{s_{0}^{*}}\left[\left(\frac{\partial f}{\partial \psi}-\frac{d}{d s} \frac{\partial f}{\partial \dot{\psi}}\right) \eta_{\psi}+\left[\frac{\partial f}{\partial r}-\frac{d}{d s} \frac{\partial f}{\partial \dot{r}}\right) \eta_{r}\right] d s \\
& -\left.\left[\frac{\partial f}{\partial \dot{\psi}} \eta_{\psi}+\frac{\partial f}{\partial \dot{r}} \eta_{r}\right]\right|_{s=0}+\left.\left[\frac{\partial f}{\partial \dot{\psi}} \eta_{\psi}+\frac{\partial f}{\partial \dot{r}} \eta_{r}\right]\right|_{s=s_{0}^{*}}+\left.\left.\left[f+\dot{r} \frac{\partial g}{\partial r}\right]\right|_{s=s_{0}^{*}} \frac{d s^{*}}{d \epsilon}\right|_{\epsilon=0} .
\end{aligned}
$$

This expression has to vanish for any admissible variation. The integral is zero if the usual EL equations are fulfilled. The boundary term at $s=0$ vanishes since $\eta_{\psi}(0)=\eta_{r}(0)=0$. Using Eq. (A7), the remainder can be brought in the form

$$
\left.\left.\left(-\dot{\psi} \frac{\partial f}{\partial \dot{\psi}}+f-\frac{\partial g}{\partial r}\right)\right|_{s=s_{0}^{*}} \frac{d s^{*}}{d \epsilon}\right|_{\epsilon=0} .
$$

This expression vanishes for arbitrary $\left.\left(d s^{*} / d \epsilon\right)\right|_{\epsilon=0}$, which corresponds to arbitrary $\eta_{\psi}\left(s_{0}^{*}\right)$ if the bracket is zero. This condition is the transversality condition ${ }^{31}$ for this variational problem. Inserting $f$ and $g$, this gives the boundary condition Eq. (3.5).

${ }^{*}$ Present address; Department of Physics, Simon Fraser University, Burnaby, British Columbia, Canada V5A 1 S6.

${ }^{1}$ See, e.g., Physics of Amphiphilic Layers, edited by J. Meunier, D. Langevin, and N. Boccara, Springer Proceedings in Physics Vol. 21 (Springer, Berlin, 1987).

${ }^{2}$ W. Helfrich, Z. Naturforsch. 28c, 693 (1973).

${ }^{3}$ H. J. Deuling and W. Helfrich, J. Phys. (Paris) 37, 1335 (1976).

${ }^{4}$ J. T. Jenkins, J. Math. Biol. 4, 149 (1977).

${ }^{5}$ S. Svetina and B. Zeks, Eur. Biophys. J. 17, 101 (1989).

${ }^{6}$ U. Seifert, K. Berndl, and R. Lipowsky, Phys. Rev. A (to be published).

${ }^{7}$ M. B. Schneider, J. T. Jenkins, and W. W. Webb, J. Phys. (Paris) 45, 1457 (1984).

${ }^{8}$ S. T. Milner and S. A. Safan, Phys. Rev. A 36, 4371 (1987).

${ }^{9}$ A. Baumgärtner and J. S. Ho, Phys. Rev. A 41, 5747 (1990).

${ }^{10}$ E. Sackmann, H. P. Duwe, and H. Engelhardt, Faraday Discuss. Chem. Soc. 81, 281 (1986).

${ }^{11}$ K. Berndl, J. Käs, R. Lipowsky, E. Sackmann, and U. Seifert, Europhys. Lett. 13, 659 (1990).

${ }^{12}$ See, e.g., H. P. Duwe, H. Engelhardt, A. Zilker, and E. Sackmann, Mol. Cryst. Liq. Cryst. 152, 1 (1987).

${ }^{13}$ U. Seifert and R. Lipowsky, Phys. Rev. A 42, 4768 (1990).

${ }^{14}$ R. Lipowsky and U. Seifert, Langmuir (to be published); Mol. Cryst. Liq. Cryst. (to be published).

${ }^{15}$ R. Lipowsky and S. Leibler, Phys. Rev. Lett. 56, 2541 (1986).

${ }^{16}$ R. Lipowsky and B. Zielinska, Phys. Rev. Lett. 62, 1572 (1989).

${ }^{17}$ M. Mutz and W. Helfrich, Phys. Rev. Lett. 62, 2881 (1989).

${ }^{18}$ N. Ostrowsky and J. Peyraud, J. Chem. Phys. 77, 2081 (1982).

${ }^{19}$ G. C. Barker and M. J. Grimson, J. Phys. (Paris) 48, 465 (1987).

${ }^{20}$ S. Leibler, R. R. P. Singh, and M. E. Fisher, Phys. Rev. Lett.
59, 1989 (1987).

${ }^{21}$ M. E. Fisher, Physica D 38, 112 (1989).

${ }^{22}$ A. C. Maggs and S. Leibler, Europhys. Lett. 12, 19 (1990).

${ }^{23}$ E. Evans, Biophys. J. 48, 175 (1985).

${ }^{24}$ M. A. Peterson, Phys. Rev. A 39, 2643 (1989).

${ }^{25}$ For $p=0$ the shape equations (2.9) with the boundary condition (3.5) can be solved for any $w>\frac{1}{2}$ in terms of elliptic functions, which allows one to verify the power laws (3.9) and (3.10) for this special case.

${ }^{26}$ The derivation of this equation is lengthy but straightforward if one used the general theory of the second variation and solves the so-called accessoric problem. An excellent presentation is given by P. Funk, Variationsrechnung und ihre Anwendung in Physik und Technik (Springer, Berlin, 1962).

${ }^{27}$ Moreover, the bound part of the vesicle contour shows oscillations which are, however, not visible on the scale of Fig. 7(a). These oscillations follow from the effective energy $F^{\text {eff }}$ for a configuration $Z(R)$ of the bound part of the vesicle, with $F^{\text {eff }}=\int d R\left[(\kappa / 2)\left(d^{2} Z / d R^{2}\right)^{2}+(\tau / 2) Z^{2}\right]$ and $\tau$ $\equiv d^{2} V /\left.d Z^{2}\right|_{Z=z_{0}}$. Typical configurations of $F^{\text {eff }}$ show damped oscillations on a length scale of the order of $\left[Z_{0}\left(\kappa / V^{0}\right)^{1 / 2}\right]^{1 / 2}$. The shorter the range or the larger the depth of the potential, the smaller the scale of these oscillations. This has previously been observed in Ref. 23.

${ }^{28}$ A. C. Maggs, D. A. Huse, and S. Leibler, Europhys. Lett. 8, 615 (1989).

${ }^{29}$ G. Gompper and T. W. Burkhardt, Phys. Rev. A 40, 6124 (1989).

${ }^{30}$ W. Helfrich and W. Harbich, Chem. Scr. 25, 32 (1985).

${ }^{31}$ See, e.g., R. Courant and D. Hilbert, Methods of Mathematical Physics (Interscience, New York, 1953), Vol. 1. 